\title{
Análisis del impacto de la innovación, las TIC y el clima empresarial sobre los ingresos de las pymes
}

\author{
Antonio Fernández-Portillo \\ Universidad de Extremadura \\ antoniofp@unex.es \\ Mari Cruz Sánchez-Escobedo \\ Universidad de Extremadura \\ maricruzse@unex.es \\ Manuel Almodóvar-González \\ Universidad de Extremadura \\ manuelag@unex.es
}

Resumen: Las organizaciones tienen como reto constante aumentar sus ingresos a través del volumen de sus ventas. Entre las formas de conseguir este objetivo encontramos la innovación y el uso de las tecnologías de la información y de la comunicación (TIC). Parte de la literatura cientifica señala que estas herramientas permiten no solo competir en el actual entorno económico, complejo y cambiante, sino también mejorar la eficiencia y la eficacia empresarial.

Por estos motivos, el objetivo de este trabajo consiste en analizar si la innovación, las TIC y el entrono repercuten en el incremento de los ingresos, a través de las ventas, teniendo en cuenta la percepción sobre el entorno que tienen los responsables de las pymes. Para estudiar el objetivo se ha realizado una encuesta a 250 pymes de la región española de Extremadura. Como aportación principal del trabajo, nuestros resultados sugieren que tanto la innovación como las TIC impactan positivamente 
en el incremento de las ventas de las pymes, $y$, por lo tanto, las empresas han de integrarlas como parte fundamental de su estructura.

Palabras clave: ventas, rendimiento empresarial, TIC, innovación, pymes, SmartPLS.

\section{Analysis of the impact of innovation, ICT and business climate on the incomes of SMES}

Abstract: Organizations face a constant challenge to increase or at least maintain their income each year through the volume of their sales,. For this reason, companies are constantly looking for different ways of meeting this challenge. Within this search process, we find the Innovation, and the use of Information and Communication Technologies (ICT) that try to improve the efficiency and efficacy of companies in this undertaking, all within a complex and constantly changing economic environment. Consequently, the present article analyzes whether innovation and ICT support the increase of income through sales, taking into account SME managers' perceptions of the environment. The article analyzes literature on the subject and surveys 250 $S M E$ f from the Spanish region of Extremadura. The main contribution of the study is that innovation and ICT have a positive impact on increasing of sales of SMEs, and therefore companies should include it as a fundamental part of their structure.

Keywords: sales; business performance; ICT; innovation; SME; SmartPLS 


\section{Introducción}

Las pymes representan uno de los factores principales de crecimiento económico y de creación de empleo (De Rassenfosse, 2012). Además, desempeñan un papel fundamental en la economía debido a que son dinámicas y a que se adaptan con flexibilidad a los cambios (Chesbrough, 2010).

Según datos del Banco Mundial, alrededor del 90\% de los negocios generados en la economía global son pymes (World-Bank, 2013). Este porcentaje se ve superado en el caso de la región de Extremadura, donde alcanza una representatividad del 99\% (INE, 2018).

En otro orden, diversas corrientes científicas reconocen el papel de la innovación como un elemento que impulsa el crecimiento y la productividad de las empresas (Kuo y Chao, 2014; World-Bank, 2015). Su incorporación en la pyme ofrece mejoras significativas en sus productos, procesos y servicios, mostrándose de manera notoria tanto en los rendimientos como en los resultados financieros $y$ económicos (Castells y Pasola, 2003; Gálvez, 2014). Por consiguiente, la innovación repercute en el entorno empresarial generando un cambio continuo. En este entorno, las pymes deben evolucionar para no ser superadas por la competencia derivada del crecimiento empresarial a nivel mundial (Gálvez, 2014; Medina y Espinosa, 1994).

Para afrontar el desafío de la competencia mediante la innovación, las empresas introdujeron las tecnologías de la información y la comunicación (TIC) (Fernández-Portillo et alii, 2019). En el caso de las pymes, su incorporación permite mejorar los procesos de innovación empresarial y, además, supone un factor de éxito a largo plazo en un entorno cada vez más competitivo (Vera et alii, 2018).

En esta línea, existe una corriente científica que analiza la relación entre las TIC y el éxito en la innovación empresarial (Fuentelsaz et alii, 2005; Gálvez, 2014; Kleis et alii, 2012). En dicha relación, encontramos investigaciones que señalan los beneficios de innovaciones en las empresas, especialmente, sobre la competitividad y la rentabilidad (Cuevas-Vargas et alii, 2016; Díaz-Chao, SainzGonzález y Torrent-Sellens, 2015; Dyerson et alii, 2009; Fernández-Portillo et alii, 2019; Gálvez, 2014; Ollo y Aramendía, 2012; Polder et alii, 2012; Vilaseca et alii, 2007).

No obstante, la mera implantación de las TIC no es suficiente para conseguir mejoras en la competitividad: aspectos como el capital humano, el crecimiento organizacional, las relaciones con los clientes y su capacidad para conseguirlos, entre otros, resultan fundamentales (Higón, 2012; Hoyos y Valencia, 2012). De hecho, es necesario equilibrar estas variables (Bigliardi y Galati, 2013; Díaz- 
Chao y Torrent-Sellens, 2010; Piget y Kossai, 2013; Spithoven et alii, 2013) para incrementar las cuotas de mercado (Norman y Verganti, 2014; Rammer et alii, 2009).

Respecto a las pymes extremeñas, caracterizadas por su ubicación rural en zonas acuciadas por procesos de despoblación, resulta fundamental el acceso a mercados exteriores si pretenden incrementar sus niveles de ventas (FernándezPortillo et alii, 2019). Según lo expuesto, la aplicación tanto de las TIC como de la innovación podría aportar una solución a este respecto, y, por esto, consideramos imprescindible conocer si realmente son medidas eficaces para generar un aumento de los ingresos por ventas, dentro de su entorno concreto. En otras palabras, es básico conocer de qué manera influye la inversión en las TIC y en la innovación en la mejora de los ingresos por ventas, y si realmente son útiles para los empresarios de zonas que comienzan a despoblarse, en aras de considerar este tipo de inversiones para la supervivencia de su empresa.

Debido a ello, surge la siguiente pregunta de investigación: ¿tienen mayores ingresos por ventas las pymes extremeñas que son innovadoras y que hacen uso de las TIC? Par tratar de dar respuesta, planteamos como objetivo estudiar si la innovación, las TIC y el entorno generan un impacto positivo sobre las ventas de las pymes.

Para analizar el objetivo, nuestro trabajo se ha estructurado de la siguiente manera: un primer apartado introductorio, en el que se aborda el objeto de investigación; un segundo apartado, donde se llevará a cabo el planteamiento del marco teórico de la investigación; a continuación, se expondrá en los apartados tercero al quinto el estudio de campo necesario para tratar de dar respuesta al objetivo, se analizarán y discutirán los resultados obtenidos, y, por último, finalizaremos con las conclusiones, limitaciones y futuras líneas de investigación, desarrolladas en los apartados sexto y séptimo, respectivamente.

\section{Marco teórico}

En este apartado expondremos las bases teóricas para el estudio de nuestro objetivo. Este, que consistía en estudiar si la innovación, las TIC y el entorno generan un impacto positivo sobre las ventas de las pymes, limita considerablemente la literatura científica, puesto que son escasas las investigaciones que estudian explícitamente la relación entre la innovación, las TIC, el entorno y el impacto de todas estas variables sobre las ventas en el marco de la pyme. No obstante, es posible señalar diversos trabajos que, de forma implícita, nos permiten conjeturar la existencia de dicha relación. 


\subsection{Las TIC y la competitividad de la pyme}

Según Valdez et alii (2016), las TIC ejercen una influencia positiva sobre las actividades de innovación, y, a su vez, esta repercute significativamente sobre la competitividad en la pyme; permitiéndole sobreponerse a sus competidores e incrementar sus cuotas de mercado (Díaz-Chao y Torrent-Sellens, 2010; Norman y Verganti, 2014; Rammer et alii, 2009). En otras palabras, las TIC mejoran el proceso de innovación (Arvanitis y Loukis, 2019) que, a su vez, genera una ventaja competitiva en el mercado (Lahi y Elenurm, 2014).

Por este motivo, si se consigue una alineación de la innovación y el uso de las TIC, se pueden lograr altos niveles de ventas (Chen y Huang, 2014; Piget y Kossai, 2013), de ingresos y de penetración de nuevos mercados (Lin y Chen, 2007). En definitiva, la cohesión entre las TIC y la innovación favorece que surjan ventajas competitivas (Alam y Mohd-Kamal, 2009; Alonso-Almeida y Llach, 2013) que incrementan las ventas y la productividad de las empresas (Apak y Atay, 2015; Kossai y Piget, 2014).

No obstante, los vínculos entre la innovación y las TIC, con su posterior repercusión en las ventas, se desarrollan dentro de un contexto. Es por esto que algunos autores destacan la relevancia de ciertos factores del entorno que actúan como elementos moderadores sobre la repercusión de innovación en la pyme (Expósito y Sanchis-Llopis, 2018; Saunila, 2017; Yang, 2017).

Tal como demuestran Ollo y Aramendía (2012), elementos del entorno, tales como la presión competitiva, la cultura nacional del país, el sistema legal o el nivel de desarrollo económico, resultan fundamentales para impulsar o detraer la adopción de las TIC en las empresas. Del mismo modo, Ghobakhloo et alii (2011) señalan ciertos factores externos (competencia, proveedores TIC y factores gubernamentales) que modelan el grado de éxito en la implantación de las TIC dentro de las pymes.

Por otro lado, el avance en conjunto de las empresas se encuentra influenciado por el entorno. En esta línea, y alineado con la teoría de los stakebolders (Ambler y Wilson, 1995; Freeman, 1984; Goodpaster, 1991; Mitchell et alii, 1997) y el modelo TOE - tecnología, organización y entorno- (Chau y Tam, 1997; Kuan y Chau, 2001; Lin y Lin, 2008; Tan et alii, 2007; Tornatzky y Fleischer, 1990; Zhu y Kraemer, 2005), es necesario considerar que para que un negocio pueda incrementar sus ventas debe existir un clima empresarial adecuado. Por esta razón el entorno empresarial juega un papel fundamental en el desarrollo de la innovación, de las TIC (Fernández-Portillo, 2016) y de los ingresos por ventas.

A la hora de estudiar el comportamiento en las pymes de la innovación y las TIC encontramos dos líneas de pensamiento. Por un lado, ciertos estudios 
sostienen que las TIC influyen en la innovación, a través del aprendizaje organizacional, transformando a las empresas en innovadoras (Petiz et alii, 2015), o bien las inversiones en TIC derivan en mejoras de productos y procesos innovadores (Arvanitis y Loukis, 2019; Díaz-Chao, Sainz-González y Torrent-Sellens, 2015).

Por otro lado, existen estudios cuyas conclusiones sugieren que la implementación de las TIC en las organizaciones, especialmente en las pymes, depende de su capacidad innovadora (Al-Ansari et alii, 2014; Fernández-Portillo et alii, 2019; Teece, 2007).

El trabajo de Ortiz-Villajos y Sotoca (2018) sugiere que el desarrollo de innovaciones es la forma más efectiva de garantizar la supervivencia de un negocio. Tal es su importancia que ciertos estudios señalan que las empresas que no innovan se enfrentan a bajos rendimientos o incluso a su disolución (Gërguri-Rashiti et alii, 2017).

La innovación desempeña un papel crucial en el éxito de la pyme (GërguriRashiti et alii, 2017), puesto que no solo mejora la competitividad, sino que incrementa la rentabilidad y los ingresos por ventas (Bigliardi y Galati, 2013; Spithoven et alii, 2013). De hecho, "las empresas innovadoras tienen mayores ventas y exportaciones" (Gunday et alii, 2011:671), y, además, "la innovación puede fomentar el crecimiento de las ventas" (Expósito y Sanchis-Llopis, 2018: 3 ).

Otros científicos se han centrado en el papel que juegan las TIC dentro de la empresa, porque representan una herramienta que impulsa la rentabilidad (Vera et alii, 2018), favorecen el crecimiento de la pyme (Rowe, 2013), y mejoran tanto la cuota de mercado como el rendimiento financiero (Prieger y Heil, 2010). Así, es posible colegir que las empresas con mayor uso de las TIC consiguen mayores niveles de ventas (Gërguri-Rashiti et alii, 2017).

\subsection{Hipótesis de estudio}

Una vez expuesto el marco teórico, y basándonos en este, procedemos a enunciar las hipótesis que vamos a contrastar empíricamente.

Hipótesis 1: El entorno empresarial ejerce un impacto positivo y directo sobre la innovación empresarial.

Hipótesis 2: El entorno empresarial ejerce un impacto positivo y directo sobre el uso de las TIC en la empresa.

Hipótesis 3: El entorno empresarial ejerce un impacto positivo y directo sobre los ingresos por ventas de la empresa.

Hipótesis 4: La innovación empresarial ejerce un impacto positivo y directo sobre el uso de las TIC en la empresa. 
Hipótesis 5: La innovación empresarial ejerce un impacto positivo y directo sobre los ingresos por ventas de la empresa.

Hipótesis 6: El uso de las TIC en la empresa ejerce un impacto positivo y directo sobre los ingresos por ventas de la empresa.

Las hipótesis planteadas se muestran en la siguiente figura, junto con el modelo teórico que se aplicará en el trabajo de campo (ver figura 1).

Figura 1. Modelo teórico con las hipótesis de la investigación

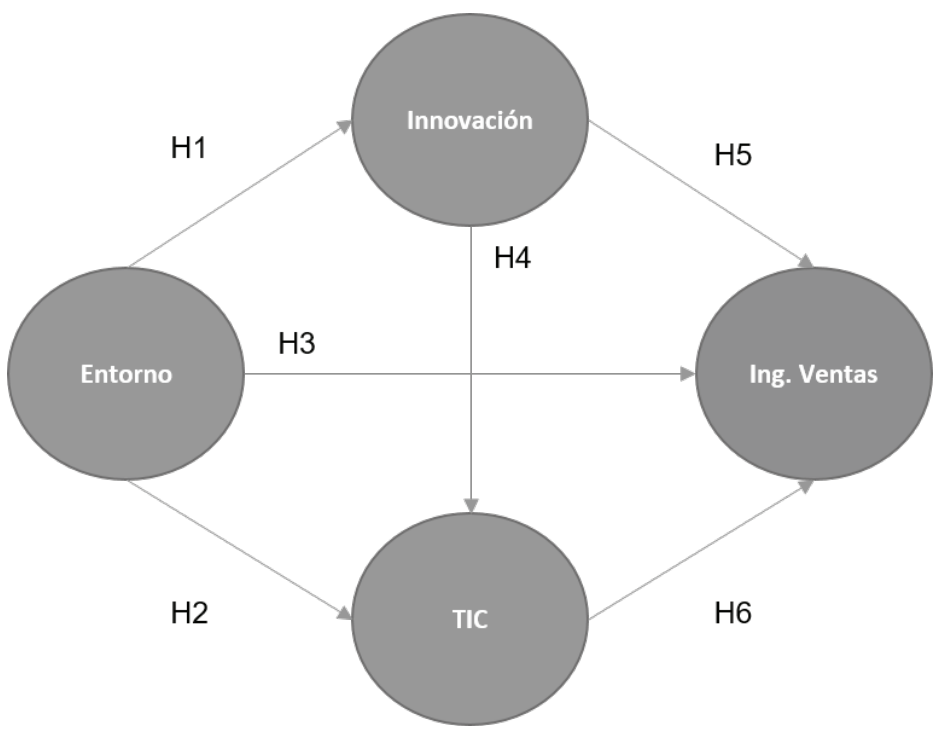

Fuente: elaboración propia.

\section{Marco empírico}

En este apartado pretendemos exponer cómo vamos a contrastar lo expuesto en el marco teórico, y así poder analizar el comportamiento que tienen las ventas de las pymes en función de su entorno, la innovación que aplican y el uso que realizan de las TIC.

Para ello, y con el fin de testar las hipótesis planteadas, se ha llevado a cabo un estudio de campo centrado en pymes mercantiles, que nos va a permitir, con posterioridad, realizar un análisis multivariante, siguiendo la metodología hipotético-deductiva.

Además, con el fin de conocer el perfil de las pymes analizadas, se ha utilizado el programa estadístico SPSS, en su versión 22.0. Para el análisis multivariante, 
nos hemos basado en el uso de modelos de ecuaciones estructurales (Structural Equations Models - SEM-), recurriendo a la técnica de mínimos cuadrados parciales (Partial Least Square, en adelante PLS), basada en la varianza, y en este caso la herramienta utilizada ha sido SmartPLS, en su versión 3.2.8.

\subsection{Diseño del estudio de campo}

Para la realización del estudio de campo se han utilizado las bases de datos del Observatorio de la Pyme de Extremadura (FAEDPYME Extremadura), teniendo en cuenta, primero, que la muestra estuviese en un espacio homogéneo, y segundo, el principio del muestreo estratificado en poblaciones finitas.

La población de empresas ha sido segmentada de acuerdo con dos criterios: actividad y comarca. Los sectores se han organizado en industria, construcción, comercio y servicios. El número de empresas en cada uno de los estratos construidos se ha obtenido a partir del Directorio Central de Empresas del INE y de los registros del Régimen General de la Seguridad Social, donde no aparecen las empresas con menos de seis asalariados.

El tamaño muestral se ha elaborado con el fin de que el error máximo fuera menor a 0,03 puntos, y un nivel de confianza del 95\%. Los datos han sido recogidos a través de encuesta telefónica, realizada por la empresa Q-Índice, realizándose test de control durante el proceso de elaboración de esta. El período de obtención de los datos fue desde febrero a abril del año 2018, siendo la muestra total obtenida de 250 pymes extremeñas.

En cuanto a las variables utilizadas para realizar nuestro estudio, nos hemos centrado en las preguntas del cuestionario, que recogen la información necesaria para conformar los constructos que pretendemos representar.

Con el fin de exponer el contenido de las variables latentes del modelo teórico propuesto (ver ), analizamos uno a uno de izquierda a derecha y de arriba abajo.

En este orden, la primera que encontramos es el entorno, un constructo que podría llegar a ser extremadamente complejo, y para el cual nos hemos centrado en medir a partir de la percepción que tienen los responsables de las pymes analizadas sobre el clima empresarial en él que se encuentra su empresa en el momento de ser encuestados.

La segunda es la innovación, variable latente que hemos construido a partir de todos los indicadores relacionados con la innovación, siguiendo los principios de la innovación marcados por Schumpeter (1942), y que hoy siguen totalmente vigentes. Las preguntas utilizadas quedan recogidas en la tabla 1. 
Tabla 1. Preguntas que conforman los indicadores que miden el constructo innovación

\begin{tabular}{|l|l|l|}
\hline ID & Preguntas & Escala \\
\hline P19_1 & Mejorar la experiencia del cliente & Likert de 5 puntos \\
\hline P19_2 & Disminuir costes & Likert de 5 puntos \\
\hline P19_3 & Aumentar la innovación en productos o procesos comerciales & Likert de 5 puntos \\
\hline P19_4 & Aumentar la innovación en procesos de dirección o gestión & Likert de 5 puntos \\
\hline P24A_1 & Cambios o mejoras en productos/servicios & No/Sí \\
\hline P24A_2 & Comercialización de nuevos productos/servicios & No/Sí \\
\hline P24A_3 & Cambios o mejoras en los procesos de producción & No/Sí \\
\hline P24A_4 & Adquisición de nuevos bienes de equipo & No/Sí \\
\hline P24A_5 & Cambios o mejoras en la organización y/o gestión & No/Sí \\
\hline P24A_6 & Cambios o mejoras en las compras y/o aprovisionamiento & No/Sí \\
\hline P24A_7 & Cambios o mejoras en comercial y/o ventas & No/Sí \\
\hline
\end{tabular}

Fuente: elaboración propia.

El tercer constructo descrito es el que hace referencia al uso de las TIC por parte de las empresas analizadas, y para su medida hemos utilizado las preguntas que mostramos en la tabla 2 .

Tabla 2. Preguntas que conforman los indicadores que miden el constructo $T I C$

\begin{tabular}{|l|l|l|}
\hline ID & Preguntas & Escala \\
\hline P16_1 & Se hacen registros manualmente en fichas o cuadernos & Likert de 5 puntos \\
\hline P16_2 & Utilizaremos software de oficina & Likert de 5 puntos \\
\hline P16_3 & Utilizaremos la nube para gestionar archivos & Likert de 5 puntos \\
\hline P17A_1 & Disponibilidad de una página web propia & No/Sí \\
\hline P17A_2 & $\begin{array}{l}\text { Realización de ventas y comercio electrónico mediante una } \\
\text { plataforma de e-commerce }\end{array}$ & No/Sí \\
\hline P17A_3 & Utilización de redes sociales con presencia activa & No/Sí \\
\hline P17A_4 & Gestión de clientes mediante plataformas CRM & No/Sí \\
\hline P17A_5 & Gestión integral de la empresa mediante un ERP & No/Sí \\
\hline
\end{tabular}

Fuente: elaboración propia.

El cuarto constructo es en el que se definen los ingresos por ventas y que medimos a través de las variables de las expectativas de ventas para el año 2018 y para 2019, y que recogemos en la tabla 3. 


\section{Tabla 3. Preguntas que conforman los indicadores que miden el constructo ventas}

\begin{tabular}{lll} 
ID & Pregunta & Escala \\
\hline P11_1 & Expectativas de ventas de su empresa para 2018 & Likert de 3 puntos \\
\hline P11_2 & Expectativas de ventas de su empresa para 2019 & Likert de 3 puntos
\end{tabular}

Fuente: elaboración propia.

\subsection{Análisis multivariante}

En esta sección nos centramos en exponer la técnica estadística utilizada para analizar las relaciones entre las variables. Respecto al análisis multivariante, este se ha llevado a cabo por las múltiples relaciones que se establecen entre los cuatro constructos expuestos, estando todos ellos interrelacionados, como hemos podido observar en el modelo teórico propuesto. Además, coincidiendo con Fernández-Portillo et alii (2015, 2019), Jiménez-Naranjo et alii (2016) y Uriel y Aldás (2005), consideramos oportuno que la técnica estadística adecuada para el estudio es la modelización estructural mediante el análisis de mínimos cuadrados parciales o Partial Least Squares (PLS).

\section{Resultados}

A continuación, vamos a mostrar los resultados obtenidos a partir del análisis de los datos utilizados en esta investigación. En primer lugar, mostraremos una tabla con los datos descriptivos de los indicadores utilizados en nuestro estudio (ver tabla 4).

Tabla 4. Valoración media de las preguntas que conforman el estudio

\begin{tabular}{|l|l|c|}
\hline ID & Pregunta & Valoración \\
\hline P11_1 & Expectativas de ventas de su empresa para 2018 & 2,38 \\
\hline P11_2 & Expectativas de ventas de su empresa para 2019 & 2,39 \\
\hline P14 & Valore de 0 (muy malo) a 10 (muy bueno) el clima empresarial actual & 6,21 \\
\hline P16_1 & No se hacen registros manualmente en fichas o cuadernos & 4,01 \\
\hline P16_2 & Utilizaremos software de oficina & 4,43 \\
\hline P16_3 & Utilizaremos la nube para gestionar archivos & 2,66 \\
\hline P17A_1 & Disponibilidad de una página web propia & 0,76 \\
\hline P17A_2 & Ventas y comercio electrónico mediante una plataforma de e-commerce & 0,19 \\
\hline P17A_3 & Utilización de redes sociales con presencia activa & 0,44 \\
\hline
\end{tabular}




\begin{tabular}{|l|l|l|}
\hline P17A_4 & Gestión de clientes mediante plataformas CRM & 0,33 \\
\hline P17A_5 & Gestión integral de la empresa mediante un ERP & 0,28 \\
\hline P19_1 & Mejorar la experiencia del cliente & 3,40 \\
\hline P19_2 & Disminuir costes & 3,56 \\
\hline P19_3 & Aumentar la innovación en productos o procesos comerciales & 3,50 \\
\hline P19_4 & Aumentar la innovación en procesos de dirección o gestión & 3,55 \\
\hline P24A_1 & Cambios o mejoras en productos/servicios & 0,68 \\
\hline P24A_2 & Comercialización de nuevos productos/servicios & 0,55 \\
\hline P24A_3 & Cambios o mejoras en los procesos de producción & 0,65 \\
\hline P24A_4 & Adquisición de nuevos bienes de equipo & 0,69 \\
\hline P24A_5 & Cambios o mejoras en la organización y/o gestión & 0,62 \\
\hline P24A_6 & Cambios o mejoras en la compras y/o aprovisionamiento & 0,60 \\
\hline P24A_7 & Cambios o mejoras en comercial y/o ventas & 0,56 \\
\hline
\end{tabular}

Fuente: elaboración propia.

\subsection{Análisis del modelo}

En este apartado, vamos a continuar con el análisis de los datos, pero desde la perspectiva del modelo propuesto. Para ello vamos a realizar el análisis multivariante, llevado a cabo, en primer lugar, el ajuste del modelo propuesto. A continuación se analizará el instrumento de medida, después, el modelo estructural propuesto, donde realizaremos el contraste de hipótesis; posteriormente, estudiaremos el efecto predictivo del modelo propuesto. Y, para finalizar, realizaremos un análisis del rendimiento de los diferentes indicadores utilizados en nuestro estudio.

\subsubsection{Análisis del ajuste del modelo}

La validación del modelo global se lleva a cabo a través del modelo FIT y del uso de los de los indicadores de bondad de ajuste, de forma que, siguiendo lo marcado por Williams et alii (2009:585), el modelo tendrá un buen ajuste cuando SRMR es menor a 0,10, o, según Hu y Bentler (1998), el SRMR sea menor a 0,08 de los resultados del modelo saturado, $y$, como podemos ver, nuestro modelo lo cumple. Además, siguiendo a Dijkstra y Henseler (2015a), se han realizado los test de ajustes exactos con la técnica del bootstraping para el modelo estimado, y de Henseler et alii (2016) se han tomado sus recomendaciones acerca de los resultados de la muestra original, para el SRMR, d_ULS y d_G sean inferiores a los valores del $95 \%$ o 99\%. En nuestro caso, podemos confirmar que los valores del modelo están dentro de lo estipulado, y estamos en condiciones de asegurar que el modelo es válido (ver ). 
Tabla 5. Validación del modelo global

\begin{tabular}{|c|c|c|c|}
\hline Modelo estimado & SRMR & d_ULS & d_G \\
\hline Muestra original & 0,038 & 0,364 & 0,132 \\
\hline $95 \%$ & 0,045 & 0,519 & 0,141 \\
\hline $99 \%$ & 0,049 & 0,615 & 0,156 \\
\hline
\end{tabular}

Fuente: elaboración propia.

\subsubsection{Valoración de modelo de medida}

Una vez comprobada la validez del modelo en relación con la muestra utilizada, procedemos a analizar los indicadores que componen nuestros constructos. Para ello procedemos a valorar los constructos en modo A, de forma que seguimos los pasos marcados por la técnica del PLS, y enumerados a continuación:

1. Fiabilidad individual del ítem.

2. Fiabilidad del constructo, de la escala o consistencia interna.

3. Validez convergente.

4. Validez discriminante.

Los parámetros que limitan cada uno de estos análisis, se encuentran recogidos en la tabla 6.

Tabla 6. Justificación de valores paramétricos

\begin{tabular}{|c|c|c|c|}
\hline Análisis & Parámetro & $\begin{array}{c}\text { Valores } \\
\text { superiores } \\
\text { a }\end{array}$ & Justificación \\
\hline $\begin{array}{l}\text { Fiabilidad } \\
\text { individual }\end{array}$ & $\operatorname{Cargas}(\lambda)$ & 0,4 & $\begin{array}{l}\text { Hair et alii (2011); Hair et alii } \\
\text { (2014) }\end{array}$ \\
\hline \multirow{3}{*}{$\begin{array}{l}\text { Fiabilidad } \\
\text { del } \\
\text { constructo }\end{array}$} & Alfa de Cronbach $(\alpha)$ & 0,7 & Nunnally y Bernstein (1994) \\
\hline & rho_A $(\rho \mathrm{A})$ & 0,7 & Dijkstra y Henseler (2015b) \\
\hline & Fiabilidad Compuesta $(\mathrm{Cr})$ & 0,6 & $\begin{array}{l}\text { Bagozzi y Yi, (1988); Nunnally } \\
\text { y Bernstein, (1994) }\end{array}$ \\
\hline $\begin{array}{l}\text { Validez } \\
\text { convergente }\end{array}$ & Varianza extraída media (AVE) & 0,5 & $\begin{array}{l}\text { Fornell y Larcker, (1981); } \\
\text { Henseler et alii (2009) }\end{array}$ \\
\hline \multirow{2}{*}{$\begin{array}{l}\text { Validez } \\
\text { discriminante }\end{array}$} & $\begin{array}{l}\text { Compara el AVE con las } \\
\text { correlaciones entre constructos }\end{array}$ & $\begin{array}{c}\text { AVE > } \\
\text { Correla- } \\
\text { ciones }\end{array}$ & $\begin{array}{l}\text { Barclay et alii (1995); Hair et alii } \\
\text { (2011); Henseler et alii (2009) }\end{array}$ \\
\hline & $\begin{array}{l}\text { Ratio heterotrait-monotrait } \\
\text { (HTMT) }\end{array}$ & 0,85 & Henseler et alii (2015) \\
\hline
\end{tabular}

Fuente: elaboración propia a partir de Fernández-Portillo (2016)

y Fernández-Portillo et alii (2019). 
Una vez expresados los parámetros que limitan cada uno de estos criterios, mostramos los resultados obtenidos en el constructo cuyos componentes están en modo A.

Tabla 7. Fiabilidad y validez del constructo

\begin{tabular}{|c|c|c|c|c|}
\hline & Alfa de Cronbach & Rho_A & $\begin{array}{c}\text { Fiabilidad } \\
\text { compuesta }\end{array}$ & $\begin{array}{c}\text { Varianza extraída } \\
\text { media (AVE) }\end{array}$ \\
\hline Ventas & 0,940 & 0,960 & 0,971 & 0,943 \\
\hline Entorno & 1 & 1 & 1 & 1 \\
\hline
\end{tabular}

Fuente: elaboración propia.

Tabla 8. Validez discriminante

\begin{tabular}{lcccc} 
& Entorno & Innovación & TIC & Ventas \\
\hline Entorno & 1,000 & & & \\
\hline Innovación & 0,326 & & & \\
\hline TIC & 0,173 & 0,523 & & \\
\hline Ventas & 0,207 & 0,285 & 0,310 & 0,971
\end{tabular}

Fuente: elaboración propia.

Para continuar con el análisis del modelo de medida, debemos analizar los constructos cuyos indicadores están configurados en modo B. Para ello, realizamos, como primer paso, un análisis de multicolinealidad, del cual nos fijaremos en el FIV (factor de inflación de la varianza), y, siguiendo a autores como Diamantopoulos y Siguaw (2006), el valor debe ser inferior a 3,3. En este paso eliminamos el indicador P24_7, con un FIV de 4,179.

Para finalizar el análisis del modelo de medida, analizaremos la relación carga-peso de los indicadores de los constructos en modo B y su significatividad, de forma que eliminaremos aquellos ítems que no sean significativos según lo estipulado por Hair et alii (2014). En este paso eliminamos el indicador P16_1, de forma que el modelo depurado ha quedado como se puede observar en la figura 2.

Una vez llegados a este punto, estamos preparados para realizar el análisis del modelo estructural, que nos va a permitir realizar el contraste de hipótesis planteado en nuestro modelo teórico. 
Figura 2. Modelo final con indicadores depurados

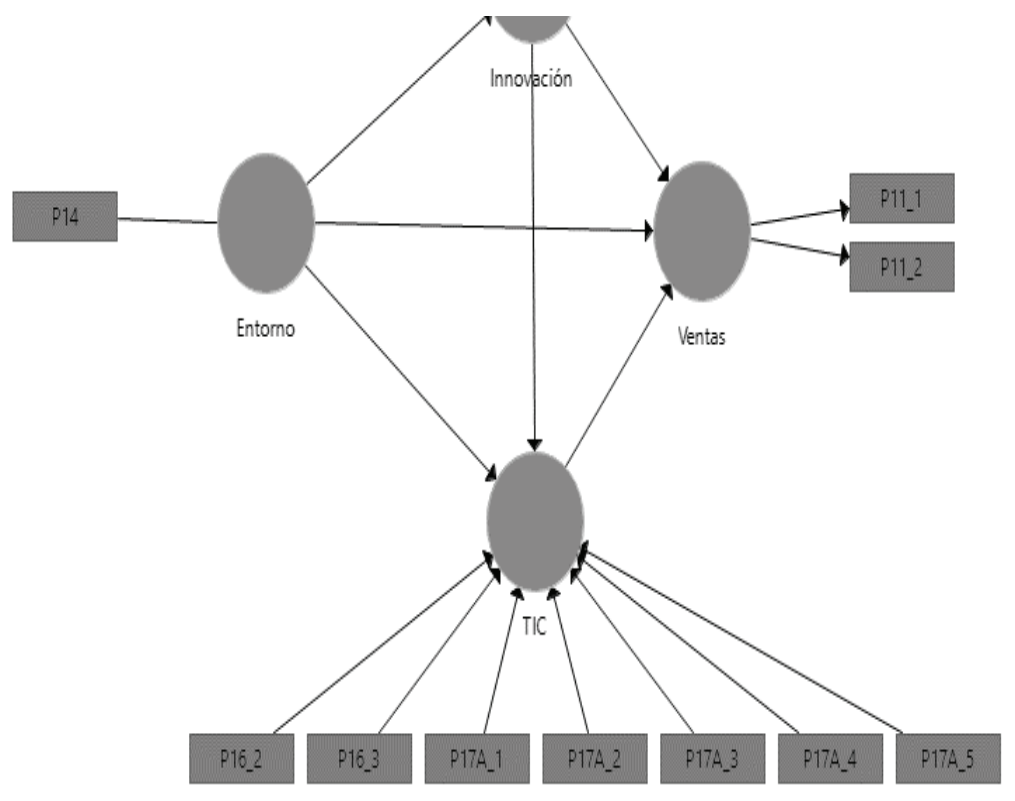

Fuente: elaboración propia.

\subsection{Análisis del modelo estructural}

A la hora de valorar el modelo estructural, debemos tener en cuenta si existen problemas de colinealidad, evaluar el signo algebraico, magnitud y significación estadística de los coeficientes path, valorar el coeficiente de determinación y su descomposición, y, por último, el tamaño de los efectos.

Siguiendo el orden mencionado anteriormente, hemos analizado la multicolinealidad entre las variables antecedentes de cada uno de los constructos endógenos (Cassel et alii, 1999), de forma que, según Hair et alii (2014: 170), existirán indicios de multicolinealidad cuando el FIV $>5$.

Tabla 9. Valoración de la colinealidad de los constructos

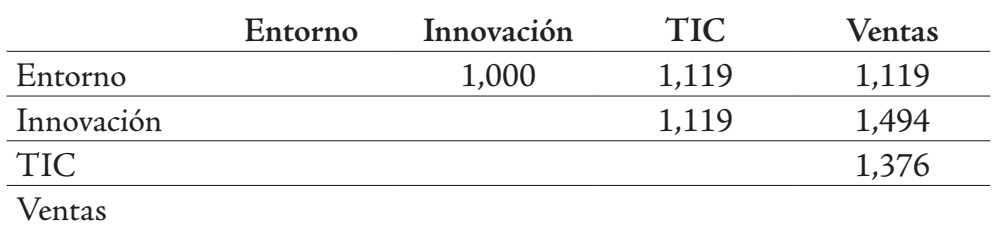

Fuente: elaboración propia. 
A continuación, evaluamos los coeficientes path de las hipótesis, de forma que, para que sean aceptadas — si la hipótesis está redactada de forma positiva—, el valor del camino debe tener el mismo signo, el intervalo de confianza no puede contener el valor cero, y el estadístico t-Student debe ser significativo para el test de una cola. En el caso de que no se cumpla alguna de estas condiciones, la hipótesis quedará invalidada.

Tabla 10. Contraste de hipótesis según sus coeficientes path

\begin{tabular}{|c|c|c|c|c|c|}
\hline \multirow[t]{2}{*}{ Hipótesis } & \multicolumn{2}{|c|}{$\begin{array}{c}\text { Intervalos de con- } \\
\text { fianza }\end{array}$} & \multirow{2}{*}{$\begin{array}{c}\text { Coeficiente } \\
\text { path }\end{array}$} & \multirow[t]{2}{*}{ Estadístico $t$} & \multirow{2}{*}{$\begin{array}{l}\text { Evaluación de } \\
\text { la hipótesis }\end{array}$} \\
\hline & $5 \%$ & $95 \%$ & & & \\
\hline $\begin{array}{l}\text { H1. Entorno } \rightarrow \text { Inno- } \\
\text { vación }\end{array}$ & 0,200 & 0,456 & 0,326 & $4,161^{* * *}$ & ACEPTADA \\
\hline H2. Entorno $\rightarrow$ TIC & $-0,108$ & 0,106 & 0,003 & $0,046 \mathrm{~ns}$ & $\begin{array}{l}\text { RECHAZA- } \\
\text { DA }\end{array}$ \\
\hline H3. Entorno $\rightarrow$ Ventas & 0,011 & 0,223 & 0,127 & 1,970 ** & ACEPTADA \\
\hline H4. Innovación $\rightarrow$ TIC & 0,447 & 0,628 & 0,522 & $9,373 * * *$ & ACEPTADA \\
\hline H5. Innovación $\rightarrow$ Ventas & 0,001 & 0,272 & 0,128 & 1,561 * & ACEPTADA \\
\hline H6. TIC $\rightarrow$ Ventas & 0,091 & 0,367 & 0,221 & $2,637 * * *$ & ACEPTADA \\
\hline
\end{tabular}

${ }^{* * *} \mathrm{p}(0,01) ;{ }^{* *} \mathrm{p}(0,05) ;{ }^{*} \mathrm{p}(0,1) ;$ ns (no significativo). t-Student de 1 cola.

Tras el contraste de las hipótesis propuestas (ver ), hemos de destacar que solo hay una hipótesis, que no está soportada por los datos utilizados en nuestro modelo, y es H2, el entorno tiene una influencia directa y positiva sobre el uso de las TIC. Además, hemos de comentar que la hipótesis 5, aunque hemos considerado que está aceptada, solo se acepta para un nivel de significación entre el 90 y el 95\%, aunque si bien es cierto es superior al 94\%. Esta hipótesis es la que hace referencia a que el uso de la innovación por parte de las empresas tiene un impacto positivo y directo sobre las ventas de la empresa.

En cuanto a la varianza explicada de las variables latentes dependientes, por los constructos que las predicen $\left(R^{2}\right)$, el valor exigido debe ser mayor a 0,1 (Falk y Miller, 1992), y, como podemos ver en la, se cumple este requisito para los tres constructos dependientes. Además, si analizamos los resultados con detenimiento, el constructo con mayor varianza explicada es el constructo TIC, con un $27 \%$, que proviene en su totalidad de la innovación. El siguiente, compuesto con mayor varianza, es ventas, que adquiere un $13,1 \%$, y que proviene en mayor medida del uso de las TIC por parte de la empresa, seguido de la innovación y, por último, de la situación del entorno. 
Tabla 11. Evaluación del nivel de $\mathrm{R}^{2}$ del modelo

\begin{tabular}{|l|c|c|c|c|c|}
\hline \multicolumn{1}{|c|}{ Hipótesis } & $\mathrm{R}^{2}$ & $\mathrm{Q}^{2}$ & Path & Correlación & Varianza explicada \\
\hline H1. Entorno $\rightarrow$ Innovación & & & 0,326 & 0,326 & $10,62 \%$ \\
\hline Innovación & 0,107 & 0,029 & & & \\
\hline H2. Entorno $\rightarrow$ TIC & & & 0,003 & 0,173 & $0,05 \%$ \\
\hline H4. Innovación $\rightarrow$ TIC & & & 0,522 & 0,523 & $27,30 \%$ \\
\hline TIC & 0,273 & 0,063 & & & \\
\hline H3. Entorno $\rightarrow$ Ventas & & & 0,127 & 0,207 & $2,62 \%$ \\
\hline H5. Innovación $\rightarrow$ Ventas & & & 0,128 & 0,285 & $3,65 \%$ \\
\hline H6. TIC $\rightarrow$ Ventas & & & 0,221 & 0,310 & $6,85 \%$ \\
\hline Ventas & 0,131 & 0,107 & & & \\
\hline
\end{tabular}

Fuente: elaboración propia.

Además, se ha realizado el análisis de la relevancia predictiva del modelo $\left(\mathrm{Q}^{2}\right)$ mediante la técnica blindfolding. En este caso es necesario, para que sea considerado un modelo de carácter predictivo, que $\mathrm{Q}^{2}$ obtenga valores mayores que 0 (Hair et alii, 2014), como sucede en este caso.

Una vez contrastado empíricamente el modelo teórico con la muestra recogida, el modelo resultante de este estudio queda representado en la figura 3.

\section{Figura 3. Modelo resultante del estudio empírico}

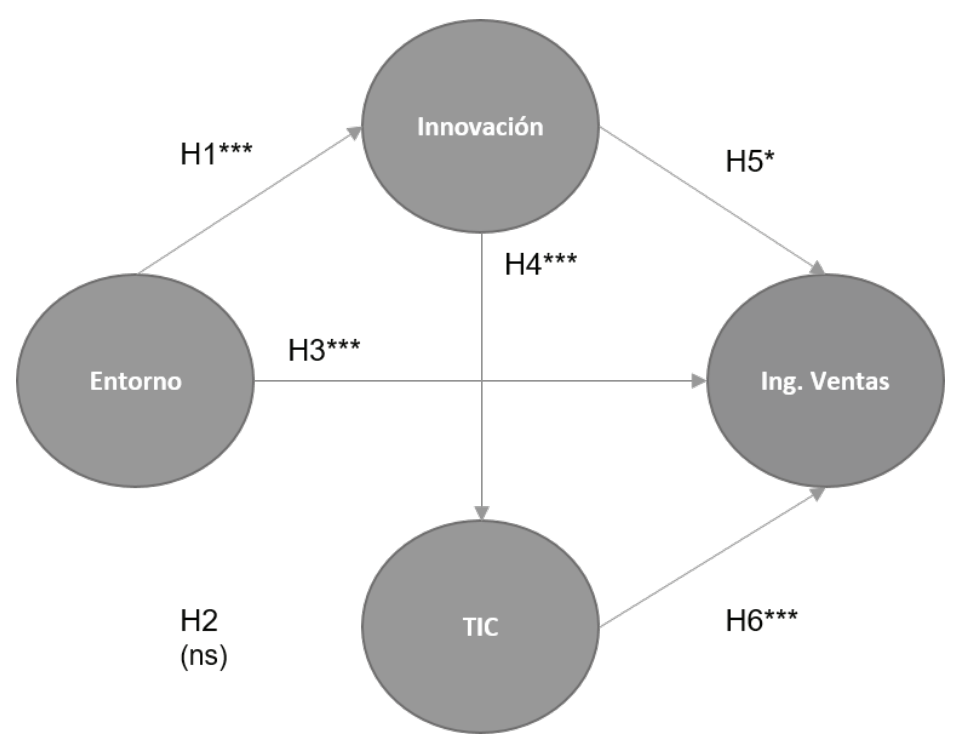

Fuente: elaboración propia. 
Para finalizar el estudio empírico hemos realizado un análisis IPMA, con el fin de conocer el rendimiento de cada uno de los indicadores utilizados, en relación con la variable exógena del modelo, en este caso, ventas. De forma que hemos obtenido los resultados que se pueden consultar en la tabla 12.

Tabla 12. Rendimiento de los indicadores sobre las expectativas de ventas

\begin{tabular}{llc} 
ID & Pregunta & Rendimientos \\
\hline P16_2 & Utilizaremos software de oficina & 85,80 \\
\hline P17A_1 & Disponibilidad de una página web propia & 76,40 \\
\hline P24A_4 & Adquisición de nuevos bienes de equipo & 69,20 \\
\hline P24A_1 & Cambios o mejoras en productos/servicios & 67,60 \\
\hline P24A_3 & Cambios o mejoras en los procesos de producción & 64,80 \\
\hline P19_2 & Disminuir costes & 64,10 \\
\hline P19_3 & Aumentar la innovación en productos o procesos comerciales & 62,50 \\
\hline P14 & Valore de 0 (muy malo) a 10 (muy bueno) el clima empresarial actual & 62,12 \\
\hline P24A_5 & Cambios o mejoras en la organización y/o gestión & 61,60 \\
\hline P19_1 & Mejorar la experiencia del cliente & 59,90 \\
\hline P24A_6 & Cambios o mejoras en la compras y/o aprovisionamiento & 59,600 \\
\hline P24A_2 & Comercialización de nuevos productos/servicios & 54,800 \\
\hline P17A_3 & Utilización de redes sociales con presencia activa & 44,400 \\
\hline P16_3 & Utilizaremos software de oficina & 41,600 \\
\hline P17A_4 & Gestión de clientes mediante plataformas CRM & 32,800 \\
\hline P17A_5 & Gestión integral de la empresa mediante un ERP & 28,400 \\
\hline P17A_2 & Ventas y comercio electrónico mediante una plataforma de e-commerce & 18,800
\end{tabular}

Fuente: elaboración propia.

Una vez expuestos los resultados de nuestro estudio, estamos en disposición de realizar la discusión de estos, tarea que realizaremos en el siguiente apartado.

\section{Discusión de resultados}

Durante la elaboración de este trabajo hemos podido dar respuesta al objetivo planteado en la introducción, obteniendo una serie de datos que resultan de interés y que vamos a desarrollar este apartado.

En primer lugar, atendiendo a la varianza explicada por el modelo teórico, es necesario destacar que el uso de las TIC supone un 6,85\% de la varianza explicada, seguido de la innovación, con 3,85\%, y, por último, el entorno, con un 2,62\%. Esto viene a corroborar lo marcado por la teoría, que destaca ampliamente a las TIC como un factor muy importante para mejorar las ventas de las pymes y la 
cuota de mercado, motivo por el cual llama especialmente la atención, que, de los indicadores de uso de las TIC en las empresas analizadas, tan solo uno de ellos tiene un despliegue superior al $45 \%$, y en este caso es la disponibilidad de una web propia.

En segundo lugar, atendiendo al rendimiento de los indicadores en relación con el constructo dependiente ventas, destacan los relacionados con el uso de las TIC, con un rendimiento del $85,8 \%$ el uso de software de oficina, $y$, con un $76,4 \%$, la disponibilidad de una página web propia, coincidiendo con la postura de $\mathrm{Ra}$ mdani y Kawaiek (2007) y Díaz-Chao y Torrent-Sellens (2010), quienes confirman que con el uso de TIC las empresas pueden llegar a mercados más amplios e internacionales incluso con recursos limitados. Además, destacan los indicadores relacionados con la innovación, como son la adquisición de nuevos bienes de equipo, con un $69,2 \%$, y cambios o mejoras en productos y/o servicios, con un $67,6 \%$. Estos resultados están alineados con lo expuesto por Expósito y Sanchis-Llopis (2018: 5) quienes afirman que "la innovación de productos causa un impacto positivo claro y significativo en el aumento de ventas", al igual que Boachie-Mensah y Acquah (2015), Díaz-Chao, Sainz-González y Torrent-Sellens (2015), Rosli y Sidek (2013) y Varis y Littunen (2010), que encontraron una relación positiva y significativa entre la innovación en productos y el rendimiento de la empresa.

En tercer lugar, en relación con el contraste de hipótesis realizado y para el caso de las pymes extremeñas, no hay relación significativa entre la percepción del clima empresarial y las ventas, en contra de lo expuesto en la teoría por Ollo y Aramendía (2012), quienes comentaban que el entorno de la empresa y la presión competitiva son un determinante importante en la adopción de las TIC en las empresas. Y lo expuesto por Baliamoune-Lutz (2003) y Mwesige (2004), quienes aseguraban que la pobreza o desarrollo económico limitado afectan negativamente la adopción de las TIC. Aunque en este segundo argumento pueda parecer que no deberían afectar a las pymes de un país desarrollado, sí debemos tener en cuenta que la región analizada destaca ampliamente por ser una zona rural, de bajo desarrollo tecnológico, y una población altamente dispersa, sin olvidar que es una de las más pobres de todo el territorio español.

En este punto, también debemos destacar que, para esta muestra, la relación entre la innovación y las ventas es aceptada con el menor nivel de significación, cuando es una de las relaciones con más apoyo teórico del modelo estudiado. Sin embargo, también es cierto que el uso de la innovación por parte de las pymes extremeñas no está del todo implantado, ya que apenas un $60 \%$ de estas son empresas innovadoras. Resultados que quizá puedan estar relacionados. De ahí que sea tan llamativo y que vaya en contra de diferentes estudios que afirman, de forma 
taxativa, que el uso de la innovación genera un aumento de las ventas (Gunday et alii, 2011); aunque, si bien es cierto, Tether y Storey (1998: 2) comentan que "el impacto en las pymes es menor que en las grandes empresas".

En cuanto al resto de hipótesis, podemos confirmar que los resultados obtenidos coinciden con lo expuesto en el marco teórico, y, por tanto, confirman que el modelo expuesto tiene carácter predictivo. Motivo por el cual podría ser utilizado para explicar resultados futuros, de los cuales se espera que, con una apuesta por la innovación y las TIC, las pymes pueden obtener una mejora significativa en sus ingresos por ventas; coincidiendo con los resultados obtenidos por otros autores como Consoli (2012), quien afirma que la inversión en TIC aumenta las ventas y la cuota de mercado.

\section{Conclusiones}

Las TIC y la innovación representan dos factores que impulsan el crecimiento de las empresas (Gálvez, 2014; Sainz-González y Torrent-Sellens, 2015; Vera et alii, 2018). Ante un entorno empresarial de despoblación rural, tal como es el caso de Extremadura (Fernández-Portillo et alii, 2019), hemos estudiado si estas herramientas, teniendo en cuenta el entorno descrito, pueden incrementar las ventas de las pymes extremeñas.

Nuestros resultados sugieren que un aumento en la inversión y la implantación de procesos de innovación conducen a un impulso de las ventas en las pymes. Efectivamente, este incremento es significativo, pero debe destacarse que no cualquier aumento en innovación se traduce en crecimiento de las ventas. En este sentido, cabe señalar que la innovación con un mayor retorno en ventas es la enfocada a los productos. Por otra parte, es necesario comentar que, de entre todas las variables estudiadas, la que genera un mayor aumento en las ventas es la que proviene de un aumento en el uso de las TIC, por lo tanto, aun conociendo las limitaciones que pueda tener la región de Extremadura, las pymes deben hacer una apuesta decidida por el uso de las TIC, con el fin de contrarrestar las pérdidas de clientes debidas a las pérdidas de población de la región que se producen cada año. Además, esto permitirá mejorar su competitividad y contrarrestar la llegada de nuevos competidores a la región.

Por último, resultan preocupantes los resultados descriptivos de los indicadores de innovación, con valores medios por debajo de un 60\%, y de TIC, por debajo del $40 \%$, en esta región, que pueden concluir con una pérdida de competitividad crítica en relación con pymes de otras regiones, tanto nacionales como internacionales. 


\subsection{Limitaciones y futuras líneas de investigación}

Debemos indicar que la limitación principal de este estudio la podemos encontrar en la forma de medir el entorno que rodea a las pymes extremeñas, y las características de esta región, ya que, aunque esté en un país desarrollado, las particularidades del entorno, las infraestructuras y la dispersión geográfica pueden afectar a los resultados.

En cuanto a las futuras líneas de investigación, sería interesante realizar un estudio comparativo o similar en las pymes de todo el país, comparando diferentes zonas con diferentes niveles de desarrollo, una comparativa entre pymes zonas rurales y urbanas, e incluso aquellas que se encuentran en áreas con alta concentración empresarial.

\section{Referencias bibliográficas}

Alam, Syed y Noor, Mohd-Kamal (2009)."ICT adoption in small and medium enterprises: An empirical evidence of service sectors in Malaysia". International Journal of Business and Management, 4 (2), 112-125.

Al-Ansari, Yahya, Jun, Xu y Pervan, Simon (2014). "A study of organizational determinants and innovation practices in Dubai SMEs". International Journal of Innovation Management, 18 (01), 1450003.

Alonso-Almeida, María del Mar y Llach, Joseph (2013). "Adoption and use of technology in small business environments". The Service Industries Journal, 33 (15-16), 1456-1472.

Ambler, Tim y Wilson, Andrea (1995). "Problems of stakeholder theory". Business Ethics: A European Review, 4 (1), 30-35.

Apak, Sudi y Atay, Erhan (2015)."Global competitiveness in the EU through green innovation technologies and knowledge production". Procedia-Social and Behavioral Sciences, 181, 207-217.

Arvanitis, Spyros y Loukis, Euripidis (2019)."Reduction of ICT Investment Due to the 2008 Economic Crisis and ICT-Enabled Innovation Performance of Firms". Journal of the Knowledge Economy, 1-27.

BAGOzzI, Richard y YI, Youjae (1988)."On the evaluation of structural equation models. Journal of the Academy of Marketing Science, 16 (1), 74-94.

Baliamoune-Lutz, Mina (2003). "An analysis of the determinants and effects of ICT diffusion in developing countries." Information Technology for Development, 10(3), 151-169. 
Bigliar Di, Barbara y Galati, Francesco (2013). "Models of adoption of open innovation within the food industry". Trends in Food Science y Technology, 30 (1), 16-26.

Boachie-Mensah, Francis y Acquah, Innocent (2015). "The effect of innovation types on the performance of small and medium-sized enterprises in the Sekondi-Takoradi Metropolis". Archives of Business Research, 3 (3).

Cassel, Claes, Hackl, Peter y Westrund, Anders (1999). "Robustness of partial least-squares method for estimating latent variable quality structures". Journal of Applied Statistics, 26 (4), 435-446.

Castells, Pere y Pasola, Jaume (2003). Tecnología e innovación en la empresa. Vol. 148. Cataluña: Univ Politèc, de Catalunya.

Chau, Patrick y Yan, Kar Yan Tam (1997). "Factors affecting the adoption of open systems: an exploratory study." MIS quarterly, 1-24.

Chen, Yue-Yang y Huang, Hui-Ling (2014). "Strategic Orientation of Knowledge Management and Information Technology and their effects on Performance". PACIS.

Chesbrough, Henry (2010). "Business model innovation: opportunities and barriers". Long range planning, 43 (2-3), 354-363.

Consoli, Domenico (2012). "Literature analysis on determinant factors and the impact of ICT in SMEs". Procedia - Social and Behavioral Sciences, 62, 93-97.

Cuevas-Vargas, Héctor, Estrada, Salvador y Larios-Gómez, Emigdio (2016). "The effects of ICTs as innovation facilitators for a greater business performance. Evidence from Mexico." Procedia Computer Science, 91, 47-56.

De Rassenfosse, Gaétan (2012). "How SMEs exploit their intellectual property assets: evidence from survey data". Small Business Economics, 39 (2), $437-452$.

Diamantopoulos, Adamantios y Siguaw, Judy (2006). "Formative versus reflective indicators in organizational measure development: A comparison and empirical illustration". British Journal of Management, 17 (4), 263-282.

Díaz-Chao, Ángel, Sainz-González, Jorge y Torrent-Sellens, Joan (2015). "ICT, innovation, and firm productivity: New evidence from small local firms." Journal of Business Research, 68 (7), 1439-1444.

Díaz-Chao, Ángel y Torrent-Sellens, Joan (2010). “¿Pueden el uso de las TIC y los activos intangibles mejorar la competitividad? Un análisis empírico para la empresa catalana." Estudios de Economía Aplicada, 28 (2), 1-22.

Dijkstra, Theo y Henseler, Jörg (2015a). "Consistent and asymptotically normal PLS estimators for linear structural equations". Computational Statistics y Data Analysis, 81, 10-23. 
Dijkstra, Theo y Henseler, Jörg (2015b). "Consistent partial least squares path modeling". MIS quarterly, 39 (2).

Dyerson, Romano, Harindranath, Gery, Barnes, David y Spinelli, Riccardo (2009). "ICT use in SMEs - A Comparison between the North West of England and the Province of Genoa". En Joaquim Filipe, David A. Marca, Boris Shishrov y Marten van Sinderen (eds.). ICE-B (pp. 244-251). INSTICC Press. Recuperado a partir de <http://dblp.uni-trier. $\mathrm{de} / \mathrm{db} /$ conf/icete/ice-b2009.html\#DyersonHBS09>.

Expósito, Alfonso y Sanchis-Llopis, Juan (2018). "Innovation and business performance for Spanish SMEs: new evidence from a multi-dimensional approach". International Small Business Journal, 36 (8), 911-931.

Falk, Frank y Miller, Nancy (1992). A primer for soft modeling. University of Akron Press.

Fernández-Portillo, Antonio (2016). Factores determinantes para la elaboración de un modelo de éxito de la empresa en el medio digital (tesis doctoral). Departamento de Economía Financiera y Contabilidad. Universidad de Extremadura.

Fernández-Portillo, Antonio, Hernández-Mogollón, Ricardo, SánChez-Escobedo, Mari Cruz y Coca-Pérez, José Luis (2019). "Does the Performance of the Company Improve with the Digitalization and the Innovation?" Annual Meeting of the European Academy of Management and Business Economics. Springer, Cham.

Fernández-Portillo, Antonio; Sánchez-Escobedo, Mari Cruz; Jiménez-Naranjo, Héctor Valentín y Hernández-Mogollón, Ricardo (2015). "La importancia de la Innovación en el Comercio Electrónico". Universia Business Review, 47, 106-125.

Foreman-Peck, James (2013). "Effectiveness and efficiency of SME innovation policy". Small Business Economics, 41 (1), 55-70.

Fornell, Claes, Larcker y David (1981). "Structural equation models with unobservable variables and measurement error: algebra and Stadistics". Journal of Marketing Research, 18 (2), 39-50.

Freeman, Edward (1988). "A Stakeholder Theory of the Modern Corporation:

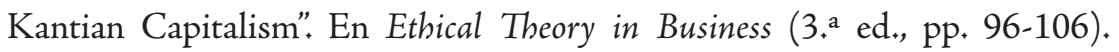
Englewood Cliffs, NJ: Prentice Hall (81).

Gálvez, Edgar (2014). "Information and Communication Technologies and Innovation in MSMEs in Colombia". Cuadernos de Administración (Universidad del Valle), 30 (51), 71-79.

Gërguri-Rashiti, Shqipe; Ramadani, Veland; Abazi-Alili, Hyrije y LéoPaul, Ratten (2017). "ICT, innovation and firm performance: the transi- 
tion economies context". Thunderbird International Business Review, 59 (1), 93-102.

Ghoвaкhloo, Morteza, Sabouri, Mohammad Sadegh, Hong, Tang Sai y Zulkifli, Norzima (2011). "Information technology adoption in Small and Medium-sized Enterprises; An appraisal of two decades literature". Interdisciplinary Journal of Research in Business, 1, 53-80.

Goodpaster, Kenneth (1991). "Business ethics and stakeholder analysis". Business ethics quarterly, 53-73.

Gunday, Gurhan, Ulusoy, Gunduz, Kilic, Kemal y Alpkan, Lutfihak (2011). "Effects of innovation types on firm performance". International Journal of Production Economics, 133 (2), 662-676.

Hair, Joseph, Hurt, Tomas, Ringle, Christian y Sarstedt, Marko (2011). "PLS-SEM: Indeed a silver bullet". Journal of Marketing theory and Practice, 19 (2), 139-152.

Hair, Joseph, Sarstedt, Marko, Hopkins, Lukas y Kuppeiwieser, Volker (2014). "Partial least squares structural equation modeling (PLS-SEM) An emerging tool in business research". European Business Review, 26 (2), 106 121.

Harindranath, Gery, Dyerson, Romano y Barnes, David (2008). "ICT adoption and use in UK SMEs: a failure of initiatives?” Electronic Journal of Information Systems Evaluation, 11 (2), 91-96.

Henseler, Jörg, Hubona, Geoffrey y Ash, Pauline (2016). "Using PLS path modeling in new technology research: updated guidelines". Industrial Management y Data Systems, 116 (1), 2-20.

Henseler, Jörg, Ringle, Christian y Sinkovics, Rudolf (2009). "The use of partial least squares path modeling in international marketing". En New challenges to international marketing (pp. 277-319). Emerald Group Publishing Limited.

Henseler, Jörg, Ringle, Christian y Sarstedt, Marko (2015)."A new criterion for assessing discriminant validity in variance-based structural equation modeling". Journal of the Academy of Marketing Science, 43 (1), 115-135.

Higón, Dolores (2012). "The impact of ICT on innovation activities: Evidence for UK SMEs". International Small Business Journal, 30 (6), 684-699.

Hoyos, Jonathan y VALEncia, Alejandro (2012)."The role of ICT in the SMES organizational environment". Revista Trilogia, 7, 105-122.

Hu, Litze y Bentler, Peter (1998). "Fit indices in covariance structure modeling: Sensitivity to under parameterized model misspecification". Psychological Methods, 3 (4), 424.

INE (2018). Instituto Nacional de Estadística, Cifras INE [www.ine.es]. 
Jiménez-Naranjo, Héctor; Coca-Pérez, José Luis; Gutiérrez Fernández, Milagros y Fernández-Portillo, Antonio (2016). "Determinants of the expenditure done by attendees at a sporting event: The case of World Padel Tour". European Journal of Management and Business Economics, 25 (3), 133 141.

Kleis, Landon; Chwelos, Paul; Ramirez, Ronald y Cockburn, Iain (2012). "Information technology and intangible output: The impact of IT investment on innovation productivity". Information Systems Research, 23 (1), 42-59.

Kossaï, Mohammed y Piget, Patrick (2014). “Adoption of information and communication technology and firm profitability: Empirical evidence from Tunisian SMEs". The Journal of High Technology Management Research, 25(1), 9-20.

Kuan, Kevin y Chau, Patrick (2001). "A perception-based model for EDI adoption in small businesses using a technology-organization-environment framework". Information y Management, 38 (8), 507-521.

Kuo, David; Chien, Liang y Chao, Chi-Yi (2014)."Exploring the relationships amongst patterns, information technology, and performance in SME-based service innovation". International Journal of Electronic Business Management, 12 (2), 102-111.

Lahi, Allan y Elenurm, Tiit (2014)."Catalysts and barriers of open innovation for SMEs in transition economy". Proceedings of the 2nd International Conference on Innovation and Entrepreneurship. ICIE: 149.

Lichtenthaler, Ulrich (2016). "Toward an innovation-based perspective on company performance". Management Decision, 54 (1), 66-87.

Lin, Carol y Chen, Mavis (2007). "Does innovation lead to performance? An empirical study of SMEs in Taiwan". Management Research News, 30 (2), 115 132.

Lin, Hsiu-Fen y Lin, Szu-Mei (2008). "Determinants of e-business diffusion: A test of the technology diffusion perspective". Technovation, 28 (3), 135-145.

López, Juan; Polo, Yolanda y Fuentelsaz, Lucio (2005). "Hacia una gestión eficaz de las tecnologías de la información”. Universia Business Review, 6, 4053.

Medina, César y Espinoza, Mónica (1994), La innovación en las organizaciones modernas. Gestión y Estrategia. México: Departamento de Administración UAM-A. 5(06).

Mitchell, Ronald; Agle, Bradley y Wood, Donna (1997). “Toward a theory of stakeholder identification and salience: Defining the principle of who and what really counts." Academy of Management Review, 22 (4), 853-886. 
Mwesige, Peter (2004). "Cyber elites: A survey of Internet café users in Uganda". Telematics and Informatics, 21 (1), 83-101.

Norman, Donald y Verganti, Roberto (2014). "Incremental and radical innovation: Design research vs, technology and meaning change". Design Issues, 30 (1), 78-96.

Nunnally, Jum y Bernstein, Ira (1994). Psychological theory. New York: McGraw-Hill.

Ollo-López, Andrea y Aramendía-Muneta, Elena (2012). "ICT impact on competitiveness, innovation and environment". Telematics and Informatics, 29 (2), 204-210.

Ortiz-Villajos, Jose y Sotoca, Sonia (2018). "Innovation and business survival: A long-term approach”. Research Policy, 47 (8), 1418-1436.

Petiz, Sara, Ramos, Fernando y Roseiro, Pedro (2015)."The use of information and communication technologies in organizational learning practices: A research study in an innovation-oriented Portuguese organization". International Journal of Advanced Corporate Learning, 8 (1), 4-11.

Piget, Patrick y Kossaï, Mohammed (2013). “The Relationship between Information and Communication Technology Use and Firm Performance in Developing Countries: A Case Study of Electrical and Electronic Goods Manufacturing SME s in Tunisia”. African Development Review, 25 (3), 330 343.

Polder, Michael, Zand, Fardad, Van-Leeuwen, George y Van-Beers, Cees (2012). "Complementarities between Information Technologies and Innovation Modes in the Adoption and Outcome Stage: A MicroEconometric Analysis for the Netherlands". CAED conference.

Pratali, Paolo (2003). "Strategic management of technological innovations in the small to medium enterprise". European Journal of Innovation Management, $6(1), 18-31$.

Prieger, James y Heil, Daniel (2010). "The microeconomic impacts of E-business on the economy". Encyclopedia of e-business development and management in the global economy (pp. 12-22). IGI Global.

Ramadani, Veland; Gërguri, Shqipe; Rexhepi, Gadaf y Abduli, Selajdin (2013). "Innovation and economic development: The case of FYR of Macedonia“. Journal of Balkan and Near Eastern Studies, 15 (3), 324-345.

Ramdani, Boumediene y Kawalek, Peter (2007)."SME adoption of enterprise systems in the Northwest of England". IFIP International Working Conference on Organizational Dynamics of Technology-Based Innovation, Boston. 
Rammer, Christian, Czarnitzki, Dirk y Spielkamp, Alfred (2009). "Innovation success of non-RyD-performers: substituting technology by management in SMEs". Small Business Economics, 33(1), 35-58.

Ratten, Vanessa (2015). "International consumer attitudes toward cloud computing: A social cognitive theory and technology acceptance model perspective". Thunderbird International Business Review, 57 (3), 217-228.

Rosenbusch, Nina, Brinckmann, Jan y Bausch, Andreas (2011). "Is innovation always beneficial? A meta-analysis of the relationship between innovation and performance in SMEs". Journal of Business Venturing, 26 (4), 441-457.

Rosli, Mohd y Sidek, Syamsuriana (2013). "Innovation and firm performance: evidence from Malaysian small and medium enterprises". XX Ibima Conference, Kuala Lumpur.

Rowe, Brian (2013). Principles of modern grinding technology. William Andrew.

Saunila, Minna (2017). "Innovation capability in achieving higher performance: Perspectives of management and employees". Technology Analysis y Strategic Management, 29 (8), 903-916.

Schumpeter, Joseph Alois (1942). Capitalism, Socialism and Democracy. New York: Harper and Brothers.

Spithoven, André, Vanhaverbeke, Wim y Roijakners, Nadine (2013). "Open innovation practices in SMEs and large enterprises". Small Business Economics, 41 (3), 537-562.

Tan, Jing, Tyler, Katherine y Manica, Andrea (2007). "Business-to-business adoption of eCommerce in China". Information and Management, 44 (3), 332 351.

Teece, David (2007). "Explicating dynamic capabilities: the nature and microfoundations of (sustainable) enterprise performance". Strategic Management Journal, 28 (13), 1319-1350.

Tether, Bruce y Storey, David (1998). "Smaller firms and Europe's high technology sectors: a framework for analysis and some statistical evidence". Research Policy, 26 (9), 947-971.

Tornatzky, Louis y Fleischer, Mitchell (1990). The process of technology innovation. Lexington (USA): Lexington Books.

Tse, Terence, Esposito, Mark y Soufani, Khaled (2016). "Fast-expanding markets: The revolution of the microeconomy". Thunderbird International Business Review, 58 (1), 5-11.

Uriel, Ezequiel y Aldás-Manzano, Joaquín (2002). Análisis multivariante aplicado. Madrid: Thomson Editores, Paraninfo SA. 
Valdez-Juárez, Luis Enrique; Ulloa, Roberto y Escobar, Elva Alicia (2016). "Las TIC, la innovación y los efectos en la competitividad de la pyme". Revista Internacional Administración y Finanzas, 9 (7), 87-108.

Varis, Miika y Littunen, Hannu (2010). "Types of innovation, sources of information and performance in entrepreneurial SMEs". European Journal of Innovation Management, 13 (2), 128-154.

Vera, Chinedu; Eze, Sunday Chinedu y Bello, Adenike Oluyemi (2018). "Actors and emerging information, communications and technology (EICT) adoption: A study of UK small and medium services enterprises". Cogent Business and Management, 5 (1), 1-19.

Vilaseca, Jordi, Torrent-Sellens, Joan, Lladós, Josep y Garay-Tamajón, Lluis (2007). "Tecnologías de la información y comunicación, innovación y actividad turística: hacia la empresa en red". Cuadernos de Turismo, (19).

Wilkinson, Thimoty y Thomas, Andrew (2014). "Innovation's second step". Thunderbird International Business Review, 56 (3), 273-284.

Williams, Larry, Vandenberg, Robert y Edwards, Jeffrey (2009). "12 structural equation modeling in management research: A guide for improved analysis". Academy of Management Annals, 3 (1), 543-604.

World-Bank. "World Bank East Asia and Pacific Economic, Update October 2013: Rebuilding Policy Buffers, Reinvigorating Growth". World Bank Publications, 2013.

World-Bank (2015)."World Bank Global Economic Prospects, Update June 2015: The Global Economy in Transition". World Bank Publications, 2015.

YANG, Judy (2017). "The governance environment and innovative SMEs". Small Business Economics, 48 (3), 525-541.

Zhu, Kevin y Kraemer, Kenneth (2005). "Post-adoption variations in usage and value of e-business by organizations: cross-country evidence from the retail industry". Information Systems Research, 16 (1), 61-84. 